\title{
BASES PARA O DESENVOLVIMENTO DE DIRETRIZES PROJETUAIS PARA O DESIGN DO MOBILIÁRIO URBANO COM APELO EMOCIONAL
}

\author{
Gabriela Zubaran Pizzato, Dra \\ UFRGS \\ gabriela.zubaran@ufrgs.br \\ Lia Buarque de Macedo Guimarães, PhD \\ UFRGS \\ liabmg@gmail.com \\ Leandro Miletto Tonetto, $\mathrm{Dr}$ \\ UNISINOS \\ Itonetto@unisinos.br
}

Resumo: A percepção de emoção de usuários na interação com o mobiliário urbano em espaços públicos destacou, em dois estudos anteriores, agradabilidade e segurança como importantes constructos emocionais. Este artigo apresenta uma revisão de literatura com objetivo de confirmar a relevância desses constructos, como base teórica para o desenvolvimento de diretrizes projetuais para o design e a avaliação do mobiliário urbano de espaços públicos com apelo emocional. Como resultado, é apresentado um quadro que relaciona atributos do produto (mobiliário urbano) e seu contexto de uso (espaço público) aos constructos pesquisados em diferentes áreas do conhecimento: design, ergonomia, psicologia ambiental, sociologia e planejamento urbano.

Palavras-chave: Design Emocional, Agradabilidade, Segurança, Mobiliário urbano, Espaço público.

Abstract: The users' perception of emotion in the interaction with street furniture in public areas highlighted in two previous studies pleasantness and safety as important constructs. This article presents a literature review in order to confirm the relevance of these constructs as theoretical basis for the development of project guidelines for the design and assessment of urban furniture in public spaces. The result is a framework that relates product attributes (urban furniture) and its context of use (public space) to constructs studied in different areas: design, ergonomics, environmental psychology, sociology and urban planning. 
Keywords: Emotional Design, Pleasantness, Safety, Urban furniture, Public space.

\section{INTRODUÇÃO}

Por definição, o mobiliário urbano engloba todos os objetos, elementos e pequenas construções integrantes da paisagem urbana, de natureza utilitária ou não, implantados mediante autorização do poder público em espaços públicos e privados (ABNT, 1986, p.1). É um produto do ambiente urbano cujo mercado atualmente é dominado por poucas grandes empresas internacionais, dotadas de forte capacidade técnica e financeira, que têm como alvo principal a exploração da publicidade acoplada ao produto (BELLINI, 2008). Brevemente resumindo, publicações relacionadas ao tema têm apresentado escopos de pesquisa com os seguintes enfoques: (i) a acessibilidade na relação entre usuário, mobiliário urbano e espaço público (ABNT, 2004; BINS ELY et al., 2006; DISCHINGER; JACKSON FILHO, 2012; BARBOSA; ALBUQUERQUE, 2010); (ii) atribuições da ergonomia como garantia de usabilidade destes elementos urbanos (BINS ELY, 1997; BESSA et al., 2002; FREITAS, 2008; LINHARES; ANJOS, 2010; VETTORETTI, 2010); (iii) necessidade de resolução de questões práticas, como, manutenção e resistência dos materiais (KINDLEIN et al., 2000; FREITAS, 2008; FERNÁNDEZ, 2010); (iv) a valorização do contexto urbano para os estudos de mobiliário urbano (MOURTHÉ, 1998; MONTENEGRO, 2005; JOHN; REIS, 2010; BASSO; VAN DER LINDEN, 2010); e, (v) aspectos formais do produto (MINGUET, 2007; SERRA, 2002). Os escopos apontados corroboram com Jordan (2000) no sentido de que, tradicionalmente, produtos são projetados com grande interesse nas questões estéticas, tecnológicas e de usabilidade. No entanto, são pouco conhecidas as emoções que podem ser evocadas a partir da interação entre usuário, ambiente e seu mobiliário urbano (BESSA, 2001; PIZZATO, et al. 2012a e 2012b).

A relação entre design e emoção tem sido explorada por profissionais do design desde os anos 90, tendo como marco inicial a Conferência Internacional sobre Design e Emoção, realizada em 1999, em Delft, Holanda, que formou uma sociedade de mesmo nome (Design and Emotion Society). O Design e Emoção, ou Design Emocional, tem como foco a compreensão do papel da emoção no uso de produtos e no desenvolvimento de técnicas e ferramentas que contribuam para a abordagem da emoção no processo de desenvolvimento de produtos. A evolução recente das publicações nesse campo enfoca a importância do desenvolvimento de projetos para o bem-estar e à felicidade (DESMET, 2012; DESMET; POHLMEYER, 2013; DORRESTIJN; VERBEEK, 2013). Pesquisas em Design e Emoção, entretanto, abordam principalmente produtos de consumo e de uso individual (PIZZATO; GUIMARÃES, 2014), ou seja, produtos industriais usados por uma determinada pessoa, que tende a desenvolver uma relação especialmente forte com o produto (LÖBACH, 2001). Tendo em vista esse cenário, acredita-se que as investigações recentes que unem a Ergonomia e o Design à emoção devam ser também direcionadas a produtos de uso coletivo, como o mobiliário urbano, podendo vir a contribuir para a maior valorização e respeito, pelos diferentes grupos de usuários na utilização do produto.

Usuários do espaço público são predominantemente desconhecidos, estranhos uns aos outros e, de certa forma, condensam traços distintivos da vida urbana (BAUMAN, 2009). Por serem utilizados por muitas pessoas, espaços públicos e seu mobiliário urbano devem agradar a maioria dos usuários (LÖBACH, 2001) para que não 
sejam vandalizados ou entrem em desuso, evitando, assim, reparos, limpeza e substituições que geram prejuízos aos cofres públicos, e contribuindo para a sustentabilidade global. Como afirma Morris (2010), o conceito de design emocionalmente durável (emotional durable design) conecta os princípios do design afetivo à sustentabilidade. Portanto, a funcionabilidade do produto e espaço urbano, a racionalidade da sua construção e forma, e a emotividade (emoções evocadas na utilização do produto) são três critérios que devem ser observados no desenvolvimento do mobiliário urbano e seu contexto de uso (CREUS, 2002).

Considerando que produtos de uso coletivo não constituem o foco do escopo de pesquisas no campo do Design e Emoção (PIZZATO et al., 2014), este trabalho apresenta uma revisão de literatura fundamentada em dois constructos emocionais: agradabilidade e segurança, a fim de verificar a relevância dos mesmos como base teórica para o desenvolvimento de diretrizes projetuais para o design e avaliação do mobiliário urbano de espaços públicos com apelo emocional.

A seleção dos constructos deve-se ao fato de ambos terem sido levantados em trabalhos que investigaram a percepção de emoção na interação entre usuários, mobiliário urbano e espaço público (PIZZATO, 2013). Esses trabalhos tiveram como objetivo gerar informação que suporte o desenvolvimento de projetos afetivos de espaços e mobiliário urbano, para torná-los mais valorizados e menos vandalizados.

\section{AGRADABILIDADE E SEGURANÇA: CONSTRUCTOS NORTEADORES}

As mais variadas emoções, positivas e negativas, podem ser experimentadas na utilização de produtos (YOON, et al., 2012; DESMET, 2008; SHEN; WYER, 2008). Além das questões ergonômicas e funcionais, relativas à função pratica dos produtos, as necessidades afetivas (função estética e função simbólica) são igualmente importantes no design de produto, mesmo sendo mais difíceis de serem comunicadas (KALID et al., 2009). Tratando-se de produtos de uso coletivo, nos quais as relações com os usuários costumam ser mais distantes do que no caso de produtos de uso individual (LÖBACH, 2001), esta dificuldade pode ser ainda maior. Sem o sentido de posse, o usuário pode ter menos interesse no produto de uso publico coletivo.

Atributos estético-simbólicos do espaço urbano também podem provocar respostas emocionais, como, prazer, excitação e relaxamento (NASAR, 1990; RUSSEL; SNODGRASS, 1987) que, por sua vez, afetam a avaliação do usuário quanto à qualidade do espaço urbano (NASAR, 1994). Dependendo de sua vitalidade, adequação, acessibilidade e o grau de controle que se tenha sobre o espaço público, ele pode ser palco de uma intensa vida urbana (LYNCH, 1981). Por outro lado, espaços públicos podem gerar emoções ruins, como medo, frustração e raiva (PIZZATO et al., 2012a e 2012b), principalmente na utilização do mobiliário urbano vandalizado, em mal estado de conservação e inadequado aos usuários.

\subsection{Agradabilidade na utilização de produtos}

A palavra agradável está associada, na língua portuguesa, ao prazer e à satisfação (HOUAISS, 2008). Neste trabalho, agradabilidade segue a abordagem do designer e criador do termo Patrick Jordan, um dos pioneiros da área a acreditar que, além de eficiência e funcionalidade, os produtos também deveriam promover experiências agradáveis a seus usuários (MONT'ALVÃO; DAMAZIO, 2008). A agradabilidade diz respeito à interação do usuário com o produto que, além de 
agradável, deve resultar em algum tipo de prazer para o usuário e lhe trazer benefícios emocionais (JORDAN, 2000).

A relação entre agradabilidade e prazer é também apontada por Jordan (2000), podendo ser entendida a partir da estrutura dos quatro prazeres, elaborada pelo antropólogo Lionel Tiger (JORDAN, 2000): prazer fisiológico: derivado dos sentidos humanos (tato, paladar, audição, olfato, e visão); prazer social: relacionado aos contatos, à imagem pessoal e ao status (encontrado em produtos e serviços que podem facilitar a interação social); prazer psicológico: relacionado às reações emocionais e cognitivas das pessoas (encontrado em objetos que são facilmente usáveis); e, prazer ideológico: relacionado ao conjunto de crenças e valores, incluindo gostos, valores morais e aspirações sociais (está relacionado à estética dos objetos e aos valores que eles englobam).

Tratando-se de um produto, a qualidade estética também está associada ao prazer que evoca da sua utilização, e envolve a combinação de formas, cores, materiais, texturas, acabamentos e movimentos, para que os produtos sejam considerados atraentes e desejáveis (IIDA, 2006). Entretanto, que a agradabilidade não é simplesmente uma propriedade de um produto, mas o resultado da interação deste com o seu usuário (JORDAN, 1998).

\subsection{Agradabilidade na utilização de espaços}

No que se referem ao espaço, estudos da Psicologia Ambiental apontam que as qualidades afetivas de um ambiente são aspectos importantes para a tomada de decisões de onde ir, retornar ou não a um determinado lugar, e podem ser utilizadas para a comparação de um lugar com outro (TUAN, 1980). Assim, espaços evocam emoções positivas e negativas, que podem ser avaliadas pelas reações emocionais e pelo significado da experiência dos usuários com o ambiente (NASAR, 1994). Espaços, públicos ou não, interferem de forma significativa na felicidade ou infelicidade das pessoas, oferecendo facilidades, como segurança, conforto, melhorias no trânsito e melhorias estéticas, ou dificuldades, opostas a todas estas citadas (SOUZA, 2003). Entretanto, a leitura dos significados relacionados ao espaço gera sensações que diferem de indivíduo para indivíduo, pois cada um percebe o espaço e relaciona-se com ele de maneira particular (TUAN, 1980). Portanto, benefícios emocionais são aqueles que compreendem como o produto interfere nas emoções das pessoas.

A avaliação estética, uma vez que é baseada em respostas emocionais, é uma avaliação afetiva (NASAR, 1998). A agradabilidade também está relacionada à qualidade estética satisfatória do espaço urbano, assim como à captação de interesse do usuário (RUSSELL, 1992; STAMPS, 2000). Para autores da Psicologia Ambiental (NASAR, 1998; STAMPS, 2000), a preferência estética envolve o ato de avaliação baseado no sentimento de prazer ou agradabilidade. As pessoas se aproximam mais de espaços agradáveis, sendo que espaços agradáveis aproximam mais as pessoas. Além disso, ao sentirem felicidade ou agradabilidade associadas ao sentimento de excitação ou entusiasmo (arousal), o espaço se torna ainda mais atrativo (RUSSEL; MEHRABIAN, 1977). Tratando-se ainda da arquitetura, um projeto de sucesso deve ser amado e mantido pelas pessoas que nele habitam, e pode ser medido em termos humanos pelo bem-estar físico e emocional dos usuários (SALÍNGAROS, 2010). KÜLLER (1980), pesquisador pioneiro nos estudos sobre arquitetura e emoção, concluiu que a agradabilidade, como regra geral, é positivamente influenciada pelas formas 
arredondadas e negativamente pelas formas quadradas. Além de ambientes agradáveis contribuírem para a calma e a segurança, reduzindo a agressividade (KÜLLER, 1980).

A relação entre emoção e formas curvas (ou orgânicas) presentes em espaços, edificações e outros produtos, como o mobiliário (Figuras 1 e 2), tem sido foco de pesquisas que apontam as seguintes conclusões: objetos lisos e arredondados conduzem a afetos positivos na sua utilização (NORMAN, 2008); formas curvas evocam sentimentos de alegria, harmonia, bem-estar, aconchego e proteção (PAPANEK, 1995); a utilização de espaços internos, com o predomínio de móveis curvos, proporciona às pessoas sentimentos de agradabilidade, calma e tranquilidade (DAZKIR; READ, 2012); as pessoas sentem-se melhor e mais livres em projetos orgânicos e bem projetados (PEARSON, 2001), pois os ambientes são mais agradáveis e reduzem o estresse (NEJAD, 2012).

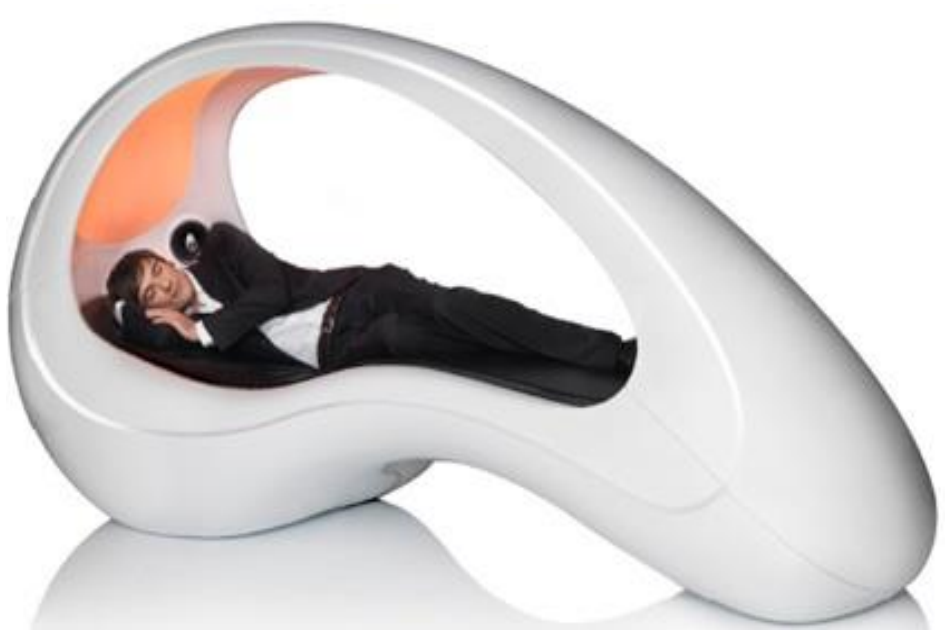

Figura 1 - Exemplo de mobiliário com linhas orgânicas desenvolvido para as práticas das "sonecas" em ambientes organizacionais. Fonte: Morris (2010)

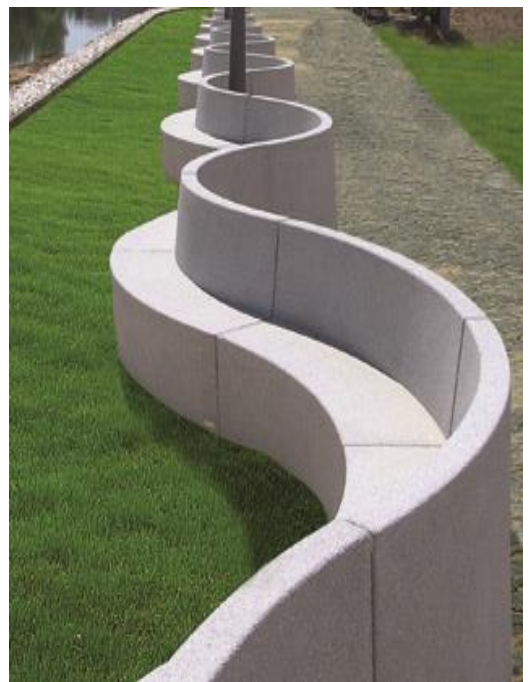

Figura 2 - Exemplo de mobiliário urbano com forma curva.

Fonte: http://urban.amop.eu

Tratando-se da avaliação de espaço, Nasar (1998, com base em Russell 1988; 1992) aponta quatro dimensões afetivas relevantes (Figura 3), entre elas a agradabilidade: (i) agradabilidade (pleasantness); (ii) potencial de atratividade (arousing); (iii) excitação (exciting); e, (iv) relaxamento (relaxing). Nas dimensões propostas pelo autor, o julgamento constitui a atitude de atribuição de valor ao ambiente/produto, enquanto que, a reação emocional, está associada à emoção evocada pelo mesmo. Já excitação e relaxamento são emocionalmente complementares à avaliação de qualidade do ambiente/objeto, e estão relacionados ao potencial de atratividade e à agradabilidade.

De acordo com Nasar (1998), o potencial de atratividade é independente da agradabilidade, ou seja, embora um ambiente possa ser ao mesmo tempo agradável e interessante, existem locais que despertam a atenção mesmo sendo caracterizados como desagradáveis. A excitação e o relaxamento referem-se à avaliação como positiva ou negativa, assim como, ao grau de interesse despertado no indivíduo por determinada composição formal: um lugar excitante, por exemplo, pode ser mais agradável e interessante do que um entediante; um lugar tranquilo é mais agradável e 
menos interessante que um entediante (NASAR, 1998). As dimensões citadas também foram aplicadas e validadas em outros estudos relacionados à avaliação de edificações e espaços urbanos (NASAR, 1994).

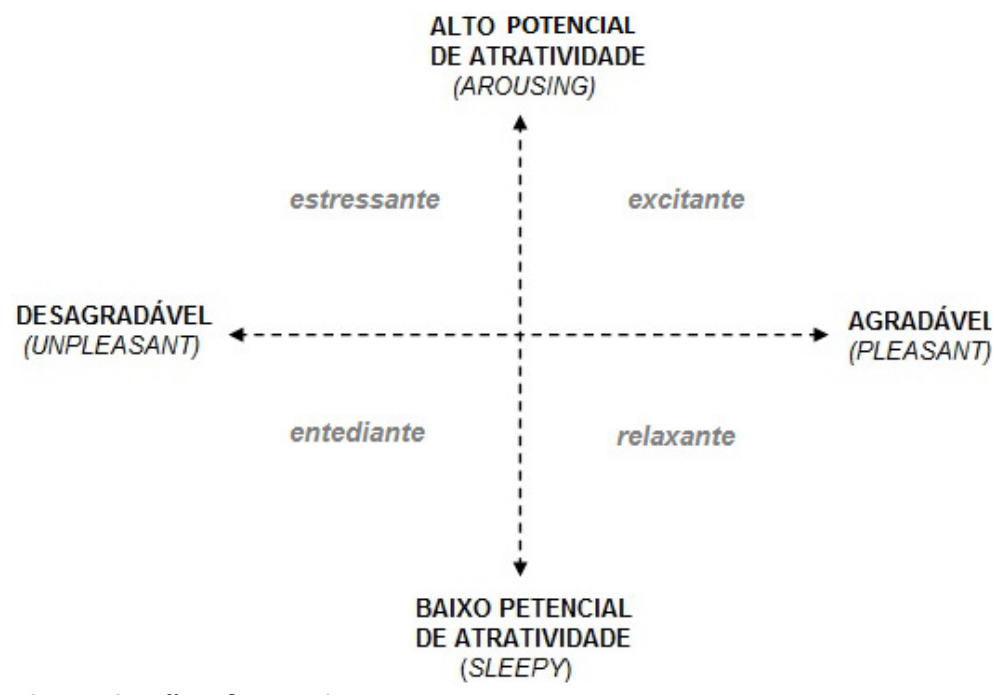

Figura 3 - Dimensões de avaliação afetiva de espaços.

Fonte: Nasar (1998), com base em Russel (1988; 1992).

\subsection{Segurança na utilização de produtos e espaços}

A segurança é uma necessidade humana, assim como a fisiológica, social, de estima e de auto realização (MASLOW, 1970). Considera-se neste trabalho a relação entre segurança e agradabilidade na utilização de produtos e espaços, visto que, sentimentos associados ao prazer na utilização de produtos estão ligados à segurança, confiança e satisfação (JORDAN, 1998). Entretanto, mesmo o ser humano estando em constante busca pela sua segurança cognitiva e emocional, em determinadas situações, a insegurança pode ser tolerada, desde que não exceda limites considerados por ele como sendo razoáveis (DIAS, 2007). Como exemplos de exceções, podem ser apontadas duas pesquisas, em especial: uma em relação ao prazer e risco na utilização de sapatos de salto (VAN DER LINDEN, 2007); e outra que trata da presença de vegetação densa em espaços, que ao mesmo tempo em que agrada a determinados usuários, evoca insegurança pela barreira visual que constitui (DESPARD, 2012).

Estudos sugerem situações favoráveis ao sentimento de segurança na utilização de espaços e produtos, incluindo o mobiliário urbano: (i) boa aparência, decorrente inclusive da manutenção (COLEMAN, 1988; REIS; LAY, 1996; NEWMAN, 1988); (ii) manutenção da vegetação (DESPARD, 2012); (iii) disposição agrupada (leiaute) de bancos que permite a socialização e incentiva o uso do espaço (FREITAS, 2008; SWART et al.,2009); (iv) acessibilidade do mobiliário urbano (PARK et al., 2013; FREITAS, 2008; BINS ELY et al., 2006; JONH, 2012) e do espaço público (BINS ELY et al., 2006; DISCHINGER; JACKSON, 2012); (v) permeabilidade visual (FREITAS, 2008); (vi) intensificação do uso do espaço por meio da presença de pessoas e de estabelecimentos com atividades variadas (JACOBS, 2011; BASSO; LAY, 2002; WHYTE, 1980; MARCUS; FRANCIS, 1998); (vii) uso de restrições no desenvolvimento do produto/espaço, que dificultam a possibilidade de erro na sua utilização (NORMAN, 2007); (viii) ausência de pichações (SPINELLI, 2012; LOUKAITOUSIDERIS, 2005); (ix) boa 
qualidade da iluminação dos espaços (IIDA, 2006; GRANDJEAN, 1998; MASCARÓ, 2008); (x) qualidade ergonômica e técnica dos produtos (IIDA, 2005); (xi) proteção a intempéries em abrigos de ônibus (BINS ELY, 1997); e, (xii) respeito ao espaço pessoal no uso do espaço público (HALL, 1996; IIDA, 2005). As mulheres, em especial, se sentem mais seguras em espaços públicos sem a presença de moradores de rua, usuários de drogas/traficantes, assediadores e assaltantes (PAIN, 2001; LOUKAITOUSDERIS, 1999; PIZZATO et al., 2012).

Tratando-se da segurança em espaços públicos, alguns autores consideram negativos os seguintes elementos: a vigilância excessiva, que gera o "espaço nervoso" e que não pode ser usado com privacidade (BAUMAN, 2009); e, elementos utilizados como barreiras (Figura 4) de proteção (septos, cercas, defensas, grades), cujo uso sem garantir permeabilidade visual pode gerar áreas de passagem sem atrativos e sem controle visual, favorecendo o vandalismo e à violência (FREITAS, 2008).

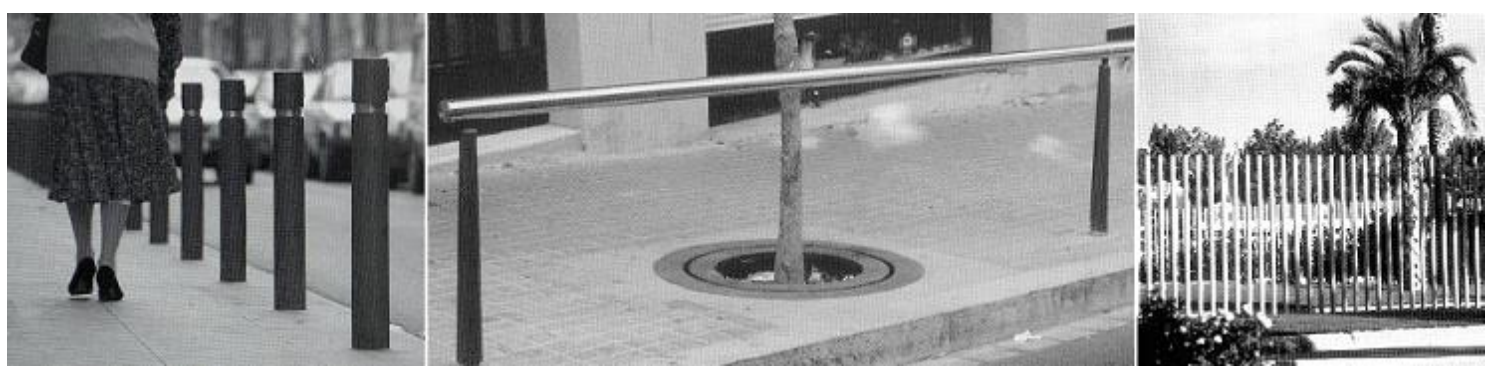

Figura 4 - Exemplo de mobiliário urbano utilizado como barreira de proteção.

Fonte: Serra (1996)

Cabe salientar que a segurança de determinados produtos e espaços é uma condição crucial (GOMES, 2003). É o caso do mobiliário urbano direcionado ao lazer de crianças de diferentes faixas etárias. A norma brasileira NBR 16071 (ABNT, 2012: partes 1 a 7) especifica os requisitos de segurança de brinquedos de uso coletivo de espaços públicos playground (balanços, escorregadores, gangorras, carrosséis, paredes de escalada, entre outros). A NBR 9050 faz referência à implantação dos elementos do mobiliário urbano para que não constituam barreiras à circulação (ABNT, 2004), e existem manuais e leis específicas de cidades do Brasil e do mundo que apontam critérios de implantação a serem seguidos (JONH, 2012).

\section{CONSTRUCTOS NORTEADORES E OS ATRIBUTOS DO PRODUTO}

Conforme o referencial teórico apresentado, os constructos agradabilidade e segurança são temas pesquisados por autores de diferentes áreas disciplinares, da psicologia ambiental à sociologia, do design ao planejamento urbano, que contribuem para estudos transdisciplinares associados à agradabilidade e segurança na utilização de produtos/espaços. A literatura revisada visa contribuir para o estabelecimento de um modelo de referencial teórico e para determinar atributos prioritários associados à agradabilidade e à segurança na utilização no mobiliário urbano e seu espaço público de uso. A Figura 5 apresenta a listagem desses atributos e suas respectivas fontes, relacionados com os constructos emocionais analisados.

A relação entre atributos (do mobiliário urbano e espaço público) e os constructos emocionais (Figura 5), identificados na revisão de literatura a qual este trabalho se propõe, demonstra que um maior número de pesquisas tem como foco a 
função prática do produto, ligada a integridade física (fisiológica/biomecânica) do usuário (LÖBACH, 2001).

\begin{tabular}{|c|c|c|}
\hline & Agr & \\
\hline 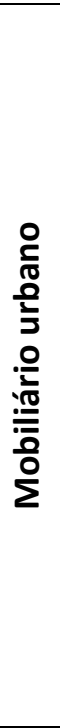 & $\begin{array}{l}\text {-linhas curvas /orgânicas (DAZKIR; READ, } \\
\text { 2012; NEJAD, 2012; NORMAN, 2008); } \\
\text {-qualidade estética (DE NISCO; WARNABY, } \\
\text { 2014; PARK et al., 2013; FREITAS, 2008; JONH; } \\
\text { REIS, 2010); } \\
\text {-emprego de materiais locais (PIZZATO et al., } \\
\text { 2013); } \\
\text {-manutenção (PARK et al., 2013; PIZZATO et } \\
\text { al. 2012; MARCUS; FRANCIS, 1990; DE NISCO; } \\
\text { WARNABY, 2014); } \\
\text {-elemento facilitador da socialização } \\
\text { (FREITAS, 2008; SWART et al.,2009, BESSA, } \\
\text { 2001 e 2002; MONTENEGRO, 2005); } \\
\text {-ordem/organização } \\
\text { (PARK et al., 2013; JONH, 2012) }\end{array}$ & $\begin{array}{l}\text {-uso de restrições no design do produto } \\
\text { (NORMAN, 2008); } \\
\text {-qualidade estética } \\
\text { (DE NISCO; WARNABY, 2014; JONH; REIS, 2010); } \\
\text { - barreiras de proteção (FREITAS,2008) } \\
\text {-atendimento a normas/leis (FREITAS, 2008; } \\
\text { GOMES, 2003; JONH; REIS, 2012); } \\
\text {-acessibilidade (PARK et al., 2013; FREITAS, } \\
\text { 2008; BINS ELY et al., 2006; JONH, 2012); } \\
\text { - implantação/leiaute do mobiliário urbano } \\
\text { (FREITAS, 2008; WHITE, 1980; FRANCIS, 1991; } \\
\text { SWART et al.,2009; PARK et al., 2013); } \\
\text {-qualidade ergonômica e técnica (IIDA, 2006) } \\
\text {-relação entre tipos de mobiliário urbano } \\
\text { (JONH; REIS, 2012); } \\
\text {-proteção a intempéries em abrigos } \\
\text { (BINS ELY, 1997). }\end{array}$ \\
\hline 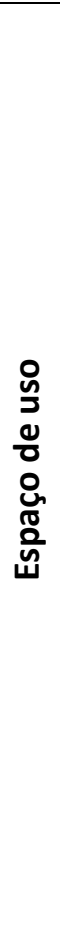 & $\begin{array}{l}\text {-presença da vegetação (PIZZATO et al. 2012; } \\
\text { DESPARD, 2012; COIRIER, 2007; JACOBS, } \\
\text { 2000; } \\
\text { STAMPS, 1997); } \\
\text {-acessibilidade (PARK et al., 2013; FREITAS, } \\
\text { 2008; BINS ELY, IIDA, 2006); } \\
\text {-qualidade estética das edificações (STAMPS, } \\
\text { 2000; MONTELLI, 2008; DE NISCO; WARNABY, } \\
\text { 2014); } \\
\text {-qualidade estética da rua, como cenário } \\
\text { urbano (NASAR, 1990; RUSSE; SNODGRASS, } \\
\text { 1987); } \\
\text {-qualidade estética da vegetação (PIZZATO et } \\
\text { al., 2012; DESPARD, 2012); } \\
\text {-ausência de pichações (SPINELLI, 2012; } \\
\text { LOUKAITOU-SIDERIS, 2005); } \\
\text {-manutenção do espaço, da vegetação e das } \\
\text { edificações (MARCUS; FRANCIS, 1990; MEIRA; } \\
\text { REIS; LAY, 2002; DESPARD, 2012; NISCO; } \\
\text { WARNABY, 2012); } \\
\text {-ausência de poluição visual (CREUS, 2002). }\end{array}$ & $\begin{array}{l}\text {-presença de pessoas e de estabelecimentos } \\
\text { com atividades variadas (JACOBS, 2000; BASSO; } \\
\text { LAY, 2002; WHYTE, 1990; MARCUS; FRANCIS, } \\
\text { 1998); } \\
\text {-acessibilidade (FREITAS, 2008; BINS ELY et al., } \\
\text { 2006; DISCHINGER; JACKSON FILHO, 2012; } \\
\text { JONH, 2012); } \\
\text {-permeabilidade visual (FREITAS, 2008) e } \\
\text { vegetação proporcionando visibilidade } \\
\text { (FREITAS, 2008; DESPARD, 2012); } \\
\text {-iluminação satisfatória (IIDA, 2006; } \\
\text { GRANDJEAN, 1998; MASCARÓ, 2008); } \\
\text {-uso e maior tempo de permanência (BESSA, } \\
\text { 2001; MARCUS; FRANCIS, 1990; GEHL, 1987; DE } \\
\text { NISCO; WARNABY, 2012); } \\
\text {-ausência de moradores de rua e pichações } \\
\text { (PAIN, 2001; LOUKAITOU-SDERIS, 1999; } \\
\text { PIZZATO et al., 2012); } \\
\text {-espaço pessoal (HALL, 1996); } \\
\text {-vigilância (BAUMAN, 2009); } \\
\text {-atendimento a normas/leis (JONH; REIS, } \\
\text { 2012). }\end{array}$ \\
\hline
\end{tabular}

Figura 5 - Relação entre atributos (mobiliário urbano e espaço público) e constructos emocionais. Fonte: Elaborado pelos autores, com base na pesquisa realizada.

O sentido de prudência e cuidado atrelados à segurança remete a atributos, como a manutenção (COLEMAN, 1988; REIS; LAY, 1996; NEWMAN, 1988), uso de restrições em projetos (NORMAN, 1997), qualidade ergonômica e técnica dos produtos (IIDA, 2005), entre outros. Por outro lado, não foram identificados atributos vinculados à função simbólica, mesmo que esta e a função estética tenham estreita relação e interdependência, e que a dimensão simbólica seja inerente a qualquer produto (LÖBACH, 2001). A manutenção da vegetação, de edificações e do mobiliário urbano, 
mesmo estando principalmente vinculada à função prática, é considerada imprescindível para a qualidade estética do espaço e de seus componentes (DESPARD, 2012).

\section{CONCLUSÃO}

Este artigo apresentou uma revisão de literatura com objetivo de confirmar a relevância de dois constructos emocionais levantados em estudos anteriores, agradabilidade e segurança. De fato, os constructos são citados em pesquisas referentes aos temas abordados, mobiliário urbano e espaço público, mas em diferentes áreas do conhecimento, como, por exemplo, design, ergonomia, psicologia ambiental, sociologia e planejamento urbano.

Os resultados demonstram que a maior parte das pesquisas identificadas são internacionais e abrangentes, sendo o contexto de uso nem sempre considerado nos estudos sobre mobiliário urbano. Por essa razão, os atributos identificados não são necessariamente os mesmos para cada um desses temas. Assim, a análise isolada de ambos pode negligenciar aspectos importantes para a garantia de segurança e agradabilidade no uso do produto e do espaço público. Por fim, ao reunir atributos do produto do espaço urbano e seu contexto de uso (espaço público), relacionando-os aos constructos abordados, este estudo contribui para a consolidação de diretrizes projetuais para o design e a avaliação do mobiliário urbano de espaços públicos com apelo emocional.

Como sugestão para trabalhos futuros, tendo como objeto de estudo o mobiliário urbano no Brasil, propõem-se: (i) a validação das relações identificadas entre construtos e atributos, apresentados na Figura 5, tomando como base a percepção de diferentes grupos de usuários; e, (ii) a exploração das relações teóricas apresentadas com a função simbólica do produto.

\section{REFERÊNCIAS}

ABNT - ASSOCIAÇÃO BRASILEIRA DE NORMAS TÉCNICAS. NBR 9283: Mobiliário Urbano. Rio de Janeiro, 1986.

ABNT - ASSOCIAÇÃO BRASILEIRA DE NORMA TÉCNICAS. NBR 9050: Acessibilidade a edificações, mobiliário, espaços e equipamentos urbanos. Rio de Janeiro, 2004.

ABNT - ASSOCIAÇÃO BRASILEIRA DE NORMA TÉCNICAS. NBR 16071: Playgrounds. Rio de Janeiro, 2012.

BARBOSA, M. B.; ALBUQUERQUE, R. Comunicação, Sinalização e Acessibilidade. In: ORNSTEIN, S.; ALMEIDA, A.; LOPES, M. (Org.). Desenho universal: caminhos da acessibilidade no Brasil. São Paulo: Annablume, 2010.

BASSO, L.; VAN DER LINDEN, J. Mobiliário urbano: origem, forma e função. In: $9^{\circ}$ CONGRESSO DE PESQUISA E DESENVOLVIMENTO EM DESIGN, 2010, São Paulo, SP.

Anais... São Paulo, 2010.

BAUMAN, Z. Confiança e medo na cidade. Rio de Janeiro: Zahar, 2009.

BELLINI, F. Abrigos de ônibus em São Paulo: análise da produção recente. Dissertação (Mestrado em Arquitetura), Faculdade de Arquitetura, Universidade de São Paulo, São Paulo, 2008.

BESSA, O. A Agradabilidade do Espaço Urbano Construído da Cidade de Alfenas (MG): uma abordagem ergonômica. Dissertação de Mestrado (Mestrado em Design), PUC Rio, 2001. 
BESSA, O.; SANTOS, E.; ROCHA, E.; MORAES, A. Ergodesign de Mobiliário Urbano a Partir de Critérios da Ergonomia do Ambiente Construído. Revista de Ergodesign e Usabilidade, v.1, n.1, p. 35-67, 2002.

BINS ELY, V. H. et al. Acessibilidade e inclusão nos espaços livres públicos. In: ENTAC (Encontro Nacional de Tecnologia do Ambiente Construído), XI, 2006, Florianópolis. Anais... ENTAC 2006. Florianópolis: ANTAC, 2006, p. 2752-2761.

BINS ELY, V. H. Avaliação de Fatores Determinantes no Posicionamento de Usuários em Abrigos de Ônibus a Partir do Método da Grade de Atributos. Tese de Doutorado (Doutorado em Engenharia de Produção) Universidade Federal de Santa Catarina, Florianópolis, 1997.

COLEMAN, A. Returning to our civilized roots. IAPS 10. Booking of Proceedings. Delft: The Netherlands, 1988.

CREUS, M. Espacios, Muebles y Elementos Urbanos. In: SERRA, Josep. Elementos urbanos, mobiliário y microarquitectura. Barcelona: Gustavo Gili, p. 6-14, 1996.

DAMAZIO, V.; MONT'ALVÃO, C. Prefácio. In: Design e Emoção, D. Norman, Rio de Janeiro: Rocco, 2008.

DAZKIR, S.; READ, M. Furniture forms and their influence on our emotional responses toward interior environments. Environment and Behavior, v. 44, n. 5, 2012.

DE NISCO, A.; WARNABY, G. Urban design and tenant variety influences on consumers' emotions and approach behavior. Journal of Business Research, v. 67, 2014.

DESPARD, E. Cultivating security: plants in the urban landscape. Space and Culture, v. 15, n. 2, p. 151-163, 2012.

DESMET, P. Product Emotion. In: SCHIFFERSTEIN, H.; HEKKERT, P. Product experience. Amsterdam: Elsevier, 2008.

DIAS, F. O Medo Social: e os vigilantes da ordem social. Lisboa: Instituto Piaget, 2007. DISCHINGER, M.; JACKSON FILHO, J. Can Tactile Tiles Create Accessible Urban Spaces? Space and Culture, v. 15, n. 3, p. 210-223, 2012.

FERNÁNDEZ, D. The design and production of urban furniture. Paisea, v. 15, 2010.

FREITAS, R. Mobiliário urbano. In: Mascaro. J. (Org), Infraestrutura da Paisagem. Porto Alegre: MaisQuatro, 2008.

GOMES FILHO, J. Ergonomia do objeto. São Paulo: Escrituras, 2003.

GRANDJEAN, E. Manual de ergonomia: adaptando o trabalho ao homem. Porto Alegre: Bookman, 1998.

HALL, E. The Hidden Dimension. New York: Doubleday \& Co, 1996.

HOUAISS, A. Dicionário de sinônimos e antônimos. São Paulo: Publifolha, 2008.

IIDA, I. Ergonomia: projeto e produção. São Paulo: Edgard Blücher, 2005.

IIDA, I. Contribuições Ergonômicas ao Design. In: 60 Congresso Internacional de Ergonomia e Usabilidade de Interfaces Humano-tecnologia: produtos, informação, ambiente construído e transporte. 2006.

JACOBS, J. Morte e vida de grandes cidades. São Paulo: Martins Fontes, 2011.

JOHN, N. Avaliação estética do mobiliário urbano e do uso de abrigos de ônibus por cadeirantes. Dissertação (Mestrado em Planejamento urbano e regional), Faculdade de Arquitetura, Universidade Federal do Rio Grande do Sul, Porto Alegre, 2012.

JOHN, N.; REIS, A. T. Percepção, estética e uso do mobiliário urbano. Gestão \& Tecnologia, v.5, p. 180-206, 2010.

JORDAN, P. Human factors for pleasure in product use. Applied Ergonomics, v. 29, n. 1, p. 25-33, 1998. 
JORDAN, P. Designing pleasurable Products: an introduction to the new human factors. London: Taylor and Francis, 2000.

KALID, H.; HELANDER, M. Customer Emotional Needs in Product Design. Concurrent Engineering: Research and Applications, v. 14, n. 3, 2006.

KALID, H. et al. Citarasa system for elicitation and analysis of affective needs. In: The Proceedings of the International Ergonomics Association 17th triennial congress, IEA 2009, 9-14 August 2009, Beijing (CD-ROM).

KINDLEIN, W.; HEILMANN, C.; AQUINO, E. Mobiliário Urbano: corrosão x design. In: CONGRESSO BRASILEIRO DE PESQUISA E DESENVOLVIMENTO EM DESIGN, n. 4, 2000, Novo Hamburgo. Anais... Novo Hamburgo: FEEVALE, 2000.

KÜLLER, R. Architecture and emotions. In: MIKELLIDES, B. Architecture for People. New York, NY: Holt, Rinehart \& Winston, p. 87-100, 1980.

LINHARES, E.; ANJOS, F. Arquitetura e ergonomia na produção de mobiliário urbano. ENGEVISTA, v. 2, n. 4, 1999.

LÖBACH, B. Design industrial: Bases para a configuração dos produtos industriais. São Paulo: Edgard Blücher, 2001.

LOUKAITOU-SIDERIS, A. Is it safe to walk here? In: TRANSPORTATION RESEARCH BOARD CONFERENCE, 2005, WASHINGTON, D.C. Proceedings....Washington, D.C:

2005. p. 102-112.

LYNCH, K. A imagem da cidade. São Paulo: Martins Fontes, 2006.

MASCARÓ, J. Infraestrutura da paisagem. Porto Alegre: Masquatro, 2008.

MARCUS, C.; FRANCIS, C. People places: design guidelines for urban open spaces. New York: Van Nostrand Reinhold, 1990.

MASLOW, A. Motivation and Personality. New York: Haper \& Row Publishers, 1970.

MINGUET, J. Arquitetura da Paisagem: mobiliário urbano. Barcelona: Monsa, 2007.

MONTENEGRO, G. A produção do mobiliário urbano em espaços públicos: o desenho do mobiliário urbano nos projetos de reordenamento das orlas do RN. Dissertação (Mestrado em Arquitetura e Urbanismo), Faculdade de Arquitetura e Urbanismo, Universidade Federal do Rio Grande do Norte, Natal, 2005.

MONT'ALVÃO, C.; DAMAZIO, V. Design, Ergonomia e Emoção. Rio de Janeiro: Mauad $\mathrm{X}, 2008$.

MOURTHÉ, Cláudia. Mobiliário Urbano. Rio de Janeiro: 2AB, 1998.

MORRIS, R. Fundamentos de Design de Produto. Porto Alegre: Bookman, 2010.

NASAR, J. Environmental aesthetics: theory, research and applications. New York:

Cambridge University Press, 1998.

NASAR, J. The evaluative image of place. In: Walsh, B; CRAIK, K; PRICE, R. Personenvironment psychology: New directions and perspectives (pp. 117-168). Mahwah, NJ: Lawrence Erlbaum, 1990.

NEJAD, M. Curvilinearity in architecture: emotional effect of curvilinear forms in interior design. Tese de Doutorado (Texas A\&M University). Texas: A\&M University. Disponível em http : / /hdl .handle .net /1969.1 /5750. Acesso em: 04 dez. 2007. NASAR, J. Urban Design Aesthetics the evaluative qualities of building exteriors.

Environment and Behavior, v.26, 1994.

NEWMAN, O. Factors influencing crime and instability in federally assisted housing developments. IAPS 10, 1988.

NORMAN, D. O design do dia a dia. Rio de Janeiro: Rocco, 2007.

NORMAN, D. Design Emocional. Rio de Janeiro: Rocco, 2008. 
PAPANEK, V. The green imperative: Natural design for the real world. New York, NY: Thames and Hudson, 1995.

PARK, S. H. et al. Design elements to improve pleasantness, vitality, safety, and complexity of the pedestrian environment: evidence from a Korean neighborhood walking case study. International Journal of Urban Sciences, 2013. Disponível em: http://dx.doi.org/10.1080/12265934.2013.776283

PEARSON, D. New organic architecture: The breaking wave. Berkeley: University of California, 2001.

PIZZATO, G. Z.; GUIMARÃES, L. B. M. Design and emotion into collective public use products? In: Proceedings of $9^{\text {th }}$ Design and Emotion conference, 2014 (artigo aprovado a ser publicado).

G. Z. Design e Emoção na utilização do mobiliário urbano em espaços

públicos. Tese de Doutorado (Doutorado em Engenharia de Produção) Universidade Federal do Rio Grande do Sul, Porto Alegre, 2013.

, G. Z.; GUIMARÃES, L. B. M.; DAMO, A. The perception of fear when using urban furniture. Work, v.41, p. 266-271, 2012.

SERRA, J. Elementos urbanos, mobiliário y microarquitectura. Barcelona: Gustavo Gili, 1996.

SHEN, H.; WYER, R. The impact of negative affect on responses to affect-regulatory experiences. Journal of Consumer Psychology, v. 18, p. 39-48, 2008.

SPINELLI, L. Pichação e comunicação: um código sem regra. Disponível em:

http://www.logos.uerj.br/PDFS/26/08lucianospen.pdf. Acesso em: out. 2012.

REIS, A.; LAY, M. C. Avaliação da qualidade de projetos - uma abordagem percebida e cognitiva. Ambiente Construído, v. 6, n. 3, 2006.

RUSSEL, J. Affective Appraisals of environments. In: NASAR, J. (Org). Environmental

Aesthetics: theory, research, and applications. New York: Cambridge University

Press, 1992.

, J.; SNODGRASS, J. Emotion and the environment. Handbook of environmental psychology, v. 1, p. 245-280, 1987. J.A.; MEHRABIAN, A. Evidence for a three-factor theory of emotions. Jornal

of Research in Personality, v. 11, p. 273-294, 1977.

SALÍNGAROS, N. Urban space and its information field. Journal of Urban Design, v. 4, n. 1, p. 29-49, 1999.

SOUZA, C. F. O espaço e a sensibilidade dos cidadãos. ARQTEXTO, v. 3, p.72-83, 2003. STAMPS, A. A psychology and the aesthetics of the built environment. USA: Kluwer Academic Publishers, 2000.

SWART, T. et al. Outdoor Seating Design to Facilitate Social Interaction Among Older Adults. Ergonomics in Design: The Quarterly of Human Factors Applications, v. 17, n. 4, p. 4-27, 2009.

TUAN, Y. Topofilia: um estudo da percepção, atitudes e valores do meio ambiente. São Paulo; Rio de Janeiro: Difel, 1980.

VAN DER LINDEN, J. Ergonomia e Design: prazer, conforto e risco no uso de produtos. Porto Alegre: UniRitter, 2007.

VETTORETTI, A. Bancos para ler e conversar: parâmetros de projeto para sistema de design generativo. Dissertação de Mestrado (Mestrado em Design) Universidade Federal do Rio Grande do Sul, RS, 2010. 
WHITE, W. The social life of small urban spaces. Washington: The conservation Foundation, 1980.

YOON, J. et al. Design for Interest: Exploratory Study on a Distinct Positive Emotion in Human-Product Interaction. International Journal of Design, v. 6, n.2, 2011. 